\title{
Agent-Based Simulation of Fish Boats Evacuation
}

\author{
Hanping Zhao ${ }^{1,2}$, Huiyan Ding ${ }^{1,2}$, Han Wang ${ }^{1,2}$ \\ ${ }^{1}$ Key Laboratory of Environment Change and Natural Disaster, Ministry of Education, Beijing 100875, China. \\ ${ }^{2}$ Academy of Disaster Reduction and Emergency Management, Beijing Normal University, Beijing 100875, China
}

Received 4 July 2015

Accepted 19 August 2015

\begin{abstract}
Emergency management agencies will organize people to evacuee when disasters are forecasted. However, contradictions between individual behavior and group objective appear frequently during large-scale evacuations, the problem is obviously in the process of fisher boats evacuation. So, this paper set up fisher boats evacuation simulation model considering individual desire of back harbor. In the agent-based model, Typhoon and harbor are inactive agents, government and fisher boats are active agents. Fisher boats make decisions whether back to harbor and which harbor to be choose according of typhoon forecast information and government instruction. The model uses the harbors of Zhejiang province as a case.
\end{abstract}

Key Words: Typhoon; fisher boat evacuation; multi-agent simulation

\section{基于主体的渔船回港避风疏散仿真 赵晗萍 ${ }^{1,2}$, 丁慧彦 ${ }^{1,2}$, 王晗 ${ }^{1,2}$}

1. 北京师范大学环境演变与自然灾害教育部重点实验室, 中国北京, 100875 ; 2. 北京师范大学减灾与应急管理研究院, 中国北京, 100875

摘要: 灾害应急管理中政府部门会根据灾害预估采取各种转移安置的措施, 但在大规模转移安置过程中对群众的安排组织工 作非常繁重, 经常会出现个体行为与集体组织出现矛盾的情况, 这种现象在台风来临前组织渔船回港避风中也非常明显。因 此在考虑渔船回港的意愿基础上设计, 设计渔船回港避风仿真模型。模型中台风与渔港作为非活动主体, 政府和渔船作为活 动主体, 渔船根据台风灾害信息、政府指令及渔港状态等要素做出是否回港和选择港口的决策。选取浙江省主要渔港作为案 例背景进行仿真实验。

关键词: 台风; 渔船疏散; 多主体仿真

1. 引言

台风灾害是中国东南沿海地区遭受的最频繁、最 严重的自然灾害之一, 平均每年有 7.2 场台风登陆中 国 $^{[1]}$ 。台风灾害破坏力极强, 台风中心风力可达 12 级 甚至更高, 台风大风往往伴随着暴雨、风暴潮等, 给 沿海居民的生产生活造成十分严重的影响。2011 年在 西北太平洋和南海共有 20 个台风生成, 其中 7 个在我 国沿海地区登陆, 共造成 23 人死亡, 7 人失踪, 直接 经济损失 192.56 亿元。2000 年以来, 台风灾害在中国
造成的年平均直接经济损失达到 318.4 亿元 ${ }^{[2]}$ 。

在台风灾害可能导致的各种损失和破坏中, 海上 作业的船只受到的影响最为直接。在中国东南沿海地 区, 海洋渔业是主导产业之一, 渔船数量众多, 目前, 中国已有海洋渔船 31.61 万艘。渔船以小型、木质渔 船为主, 钢制、大型渔船较少, 旧渔船多, 新造渔船 少, 船龄普遍偏高, 10 年以上船龄的渔船占 $60.8 \%{ }^{[3]}$, 渔船本身安全性较低。因此, 在台风灾害应急响应中, 
及时有效的渔船回港避风是一个不容忽视的终于环节。

在应急疏散中涉及人员众多, 难以在短时间内让 大规模群众完全听从指挥, 极易出现个体按照自我意 愿自主行动的现象。因此在应急中应充分考虑个体行 动的意愿和偏好, Helbing 等人 (2000) $)^{[4]}$ 在 《Nature》 上发表了研究成果, 通过人群在恐悅状态下的行为构 建了恐慌状态下的人群逃散模型。Hao-Che Wu 等 (2012) ${ }^{[5]}$ 在美国卡特里娜和丽塔䢐风之后以调查问 卷的形式分析疏散时人们的路径选择行为。胡红等 (2007) ${ }^{[6]}$ 根据将驾驶员对突发事件的心理行为反应 调查, 对 GIPPS 跟驰模型进行了改进, 建立了车速与 安全车距的关系模型。HuizhaoTu 等 (2010) ${ }^{[7]}$ 为研究 疏散人员的驾驶行为对整个疏散过程的作用, 采用 Paramics 微观仿真软件对疏散人员的驾驶行为进行不 同情景的模拟仿真, 研究车辆加速度、最大行驶速度、 平均距离、最小间距等因素对仿真结果的影响。Stern 等人 (1989) ${ }^{[8]}$ 利用行为模拟研究了不同类型家庭的 行动 (外出、在家或睡觉) 为整体预警信息传播的影 响。Uchida (2012) ${ }^{[9]}$ 研究了在滑坡与预警中人群疏散 概率变化的现象。

考虑个体行为因素后, 系统建模则多数采用基于 多主体仿真 (Agent-Based Simulation: ABS) 手段模拟 人群疏散行为。ABS 是一种微观仿真技术, 仿真模型
中主体具有自主性和目标性, 可以与环境和其他主体

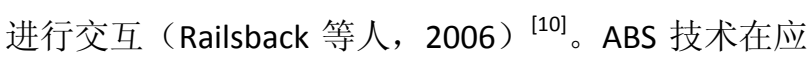
急响应建模中已经有了广泛的应用, Chen 和 Zhan (2008) ${ }^{[11]}$ 研究了城市交通疏散模型; Banerjee 等 (2009) ${ }^{[12]}$ 针对大规模拥堵问题建立仿真模型; Magesh 等 (2012) ${ }^{[13]}$ 提出基于 ABS 的预警信息扩散 下疏散仿真的框架, 其中利用概率原理模拟了疏散中 警示邻居的行为。Hui 等人 (2008) ${ }^{[14]}$ 利用 ABS 模型 研究个体行为如何影响预警信息如何在社区间传播。

对于渔船疏散问题，在台风来临时候管理渔船回 港避风时候更加明显, 渔民倾向于在海上尽量多停留 可以多打些鱼，但同时面临的被台风打击的风险也会 随之增加。渔民在海上相对分散, 很难进行有效组织 管理, 因此本文针对渔民疏散问题进行研究, 在对渔 民回港避风意愿调研基础上, 设计了基于主体的渔船 回港避风仿真模型。

\section{2. 渔船回港基于主体自主决策仿真建模}

2.1 渔船回港仿真结构

在台风灾害渔船回港避风的问题中, 有政府、渔 船、台风和港口 4 个要素, 它们组成了一个互动的灾 害应急响应系统。仿真的结构与给要素之间的关系, 如图 1 所示:

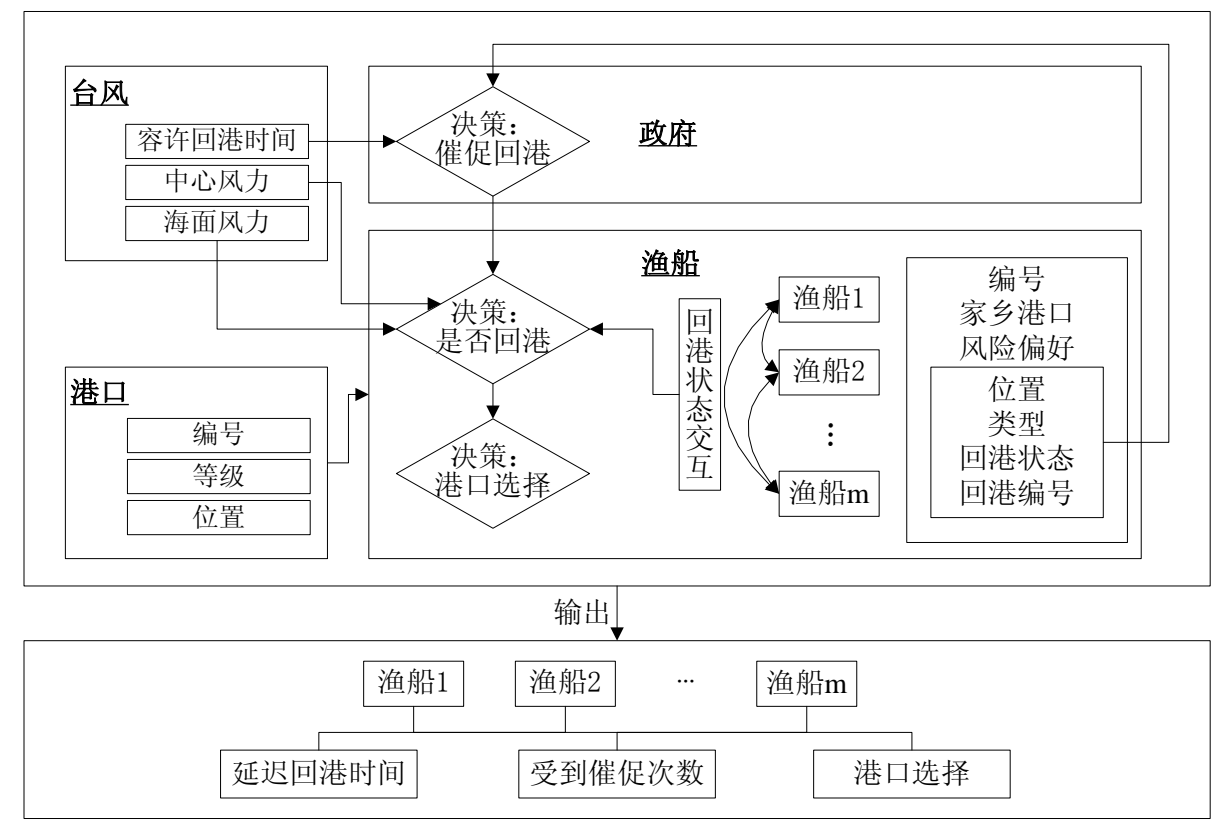

图 1. 基于主体自主决策仿真结构 
政府可以获得台风的预报和预警信息，通过对渔 船和港口情况的监测, 监督渔船回港避风, 做出“催促 回港”的决策, 在系统中只有一个。渔船同样可以得到 台风的预警信息, 并能感知自身所处位置气象信息, 根据对于自身台风风险状况的认知, 和政府的相关指 令, 做出“是否回港”及“港口选择”的决策, 在系统中 有多个。政府获得渔船的位置信息和回港状况, 渔船 则对政府“督促回港”的指令的反应。

台风具有风级、路径、等属性, 港口具有等级、 容量等属性, 它们的状态都会随时发生变化, 它们是 仿真的外部环境。渔船和政府会根据它们的变化而做 出决策, 而渔船回港状态的改变, 也会对港口产生影 响, 引起其容量属性的变化。

\section{2 渔船回港仿真主体要素设计}

\subsection{1外部环境主体}

外部环境是非活动的主体，人在短时间内无法干 预和改变的主体, 但外部环境状态会影响活动主体的 决策。台风和港口具体属性设置如表 1 所示。大风预 报图进行模拟见图 2。

\subsection{2 活动主体行为定义}

活动主体具有自主决策能力, 它可以感知外界信 息并做出决策。对于不同的主体，可以感知的信息和 决策内容如表 2 所示。

\subsection{3 渔船回港决策行为模式}

2011 年 6 月 14 日-17 日, 在浙江省岱山县就渔船

表 1. 台风和港口属性列表

\begin{tabular}{|c|c|}
\hline 主体名称 & 属性 \\
\hline \multirow[b]{3}{*}{ 台风 } & $\begin{array}{l}\text { 容许回港时间 } \boldsymbol{T} \text { ：根据台风预报信息，估计台风到达的时间，规定所有应急活动需在该时间 } \\
\text { 内完成 }\end{array}$ \\
\hline & 台风中心风力 $W c:$ 预报时刻台风中心的风力等级 \\
\hline & $\begin{array}{l}\text { 海面任意点风力预报 } W_{i} \text { : 气象部门对于海面大风情况的预报。在这里, 使用气象部门发布的 } \\
\text { 大风预报图进行模拟 (图 2)。对于预报时段内的任意时刻, 海面上任意点的风力值服从正 } \\
\text { 态分布。在 } 10 \text { 级风圈内时: 均值为 }(10+W c) / 2 \text {, 标准差满足 } 6=W c-10 \text {; 在 } 7 \text { 级风圈内时: 均 } \\
\text { 值为 } 8.5 \text {, 标准差为 } 0.5 \text {; 在 } 7 \text { 级风圈外时: 均值为 } 3.5 \text {, 标准差为 } 1.17\end{array}$ \\
\hline \multirow{3}{*}{ 港口 } & 港口编号：可提供避风的港口编号 \\
\hline & 港口等级 $\boldsymbol{l}$ : 对于不同的港口等级, 具有不同的可容纳渔船类型集合 $\left\{H S_{l}\right\}$ \\
\hline & 港口位置: 由坐标 $x$ 和 $y$ 来表示港口的位置 \\
\hline
\end{tabular}

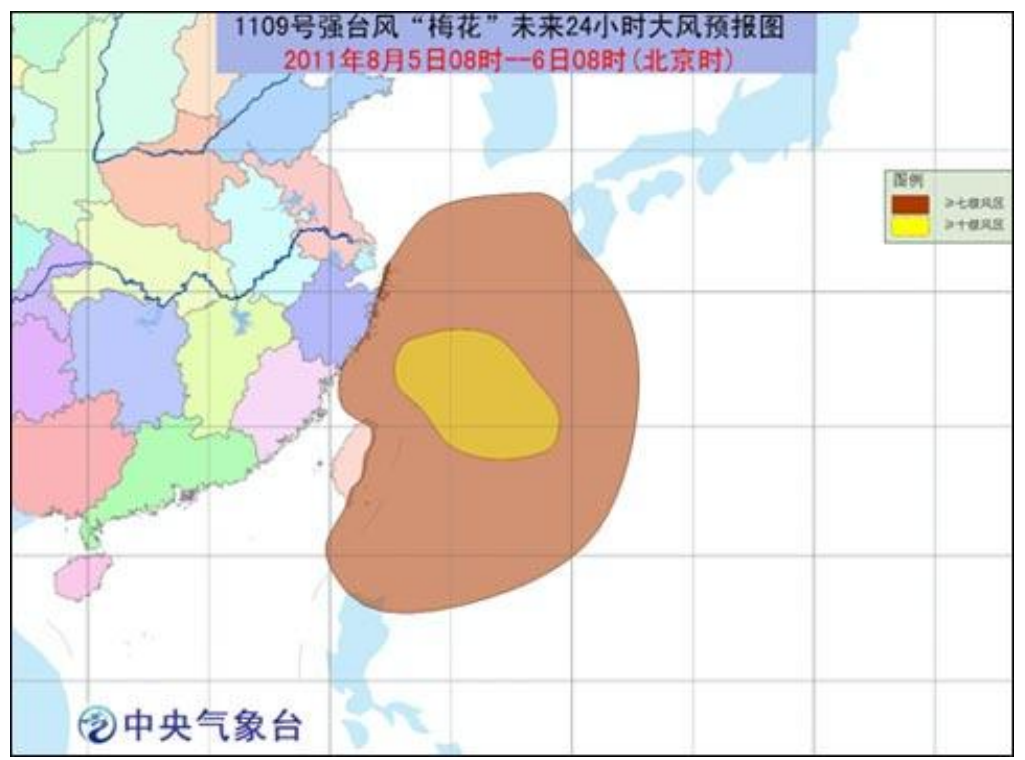

图 2. 中央气象台台风大风预报图（来源: 中国天气网[68]）

Published by Atlantis Press Copyright: the authors 
表 2. 政府和渔船的属性、感知信息和决策内容

\begin{tabular}{|c|c|c|}
\hline 主体名称 & 属性 & 内容 \\
\hline \multirow{2}{*}{ 政府 } & $\begin{array}{l}\text { 感知 } \\
\text { 信息 }\end{array}$ & $\begin{array}{l}\text { 台风: 容许回港时间 } T \text { （台风预计登陆的时间）; } \\
\text { 港口: 港口的位置（坐标 } x \text { 和 } y \text { ); } \\
\text { 渔船：渔船的位置（坐标 } x \text { 和 } y \text { ），回港状态 } I b_{i} \text {, 回港编号 } S h_{i} \text {, 渔船类型 } k \text { 。 }\end{array}$ \\
\hline & $\begin{array}{l}\text { 决策 } \\
\text { 内容 }\end{array}$ & $\begin{array}{l}\text { 政府主要通过与渔船的交互, 做出是否催促该渔船回港的决策。政府根据渔船回港需 } \\
\text { 要的时间, 当渔船的回港时间 } t_{i} \text { 超过剩余容许回港时间 } T_{r} \text { 的一半时, 决定对该渔船“催 } \\
\text { 促回港” }\end{array}$ \\
\hline \multirow[t]{3}{*}{ 渔船 } & 属性 & 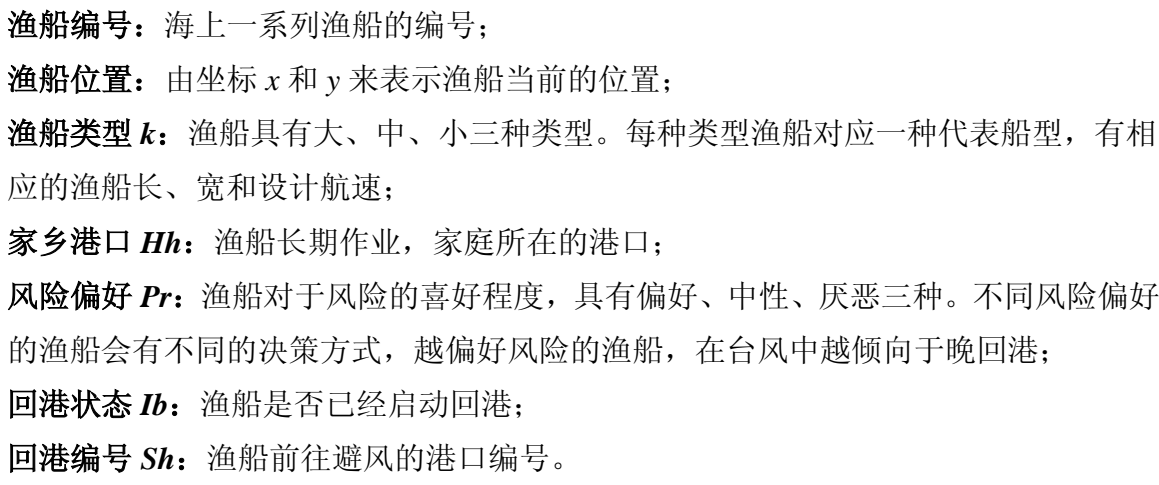 \\
\hline & $\begin{array}{l}\text { 感知 } \\
\text { 信息 }\end{array}$ & $\begin{array}{l}\text { 台风: 容许回港时间 } T \text { (预计登陆的时间), 台风中心风力 } W c \text {, 自身所处海面的风力 } \\
W ; \\
\text { 港口: 港口的位置（坐标 } x \text { 和 } y \text { ），可容纳渔船类型 }\left\{H S_{l}\right\} ; \\
\text { 政府：政府“催促回港”的命令; } \\
\text { 渔船：距自身一定半径 } R \text { 内渔船的是否回港决策。 }\end{array}$ \\
\hline & $\begin{array}{l}\text { 决策 } \\
\text { 内容 }\end{array}$ & $\begin{array}{l}\text { 渔船根据外部环境和政府信息, 做出是否回港和港口选择的决策。具体决策准则在 } \\
\text { 1.2.3.小节阐述 }\end{array}$ \\
\hline
\end{tabular}

回港避风中渔民的决策行为因素进行了调研。综合调 研中从乡镇管理渔业管理部门和渔民两方面了解的结 果, 可以看到, 目前渔船在回港避风中, 政府管理部 门更多承担的是“监督”的角色, 即保证在渔船在一定 的时间内进入港口。但在回港的具体行动上, 是由渔 船自主决策的, 主要有如下方面:

1. 回港时机：渔船根据的台风风级、走向等气象信 息, 回港需要的时间, 政府监督情况及经济收益 等因素, 判断开始回港的具体时刻。

2. 港口选择：根据回港需要的时间, 判断是“回家” 避风, 还是“就近”避风。

（1）是否回港

渔船当前时刻是否回港的决策, 主要基于渔船根 据风力对自身风险情况的判断, 及自身风险偏好 $P r_{i}$ 情况来决定, 是渔船基于风险评估的应急响应决策。 根据调研情况，将渔民风险认知的因素简单设定为台
风中心风力 $W c$ 和自身所处海面的风力 $W_{i}$ 。渔船根据 台风中心风力 $W c$ 判断此次台风的破坏程度, 根据自 身所处海面的风力 $W_{i}$ 判断自己当前状况的危险程度。

渔民根据台风风力的情况, 而做出的回港决策处 理成一种模糊逻辑关系，即：对于不同的风险偏好类 型的渔民，在不同的台风情况下，以一定的概率会决 定回港。在不同情况下，决定回港的概率如表 3 和 4 所示。表中的参数均根据调研情况假设得到。

表 3 给出了在不同台风中心风力 $W c$ 情况下, 判 断自己的理论回港时间 $t_{i}$ 提前容许回港时间 $T$ 的小时 数 $t^{\prime}{ }_{i}$, 在不同的提前程度 $t^{\prime}$ 下, 决定回港的概率 $P b_{i}$ 。 如表中带下划线的数字表示: 在台风中心风力为 9 级 时, 若风险厌恶型渔船的需要回港时间在 24 小时到 12 小时之间, 有 0.1 的概率选择回港。 
表 3. 根据台风中心风力 渔船回港概率表

\begin{tabular}{|c|c|c|c|c|c|c|c|c|c|c|c|c|c|c|c|c|}
\hline 中心风力 & \multicolumn{4}{|c|}{$<10$ 级 } & \multicolumn{4}{|c|}{10 级 } & \multicolumn{4}{|c|}{11 级 } & \multicolumn{4}{|c|}{12 级 } \\
\hline 提前时间 & 24 & 12 & 8 & 4 & 24 & 12 & 8 & 4 & 24 & 12 & 8 & 4 & 24 & 12 & 8 & 4 \\
\hline 风险厌恶 & 0 & 0 & 0 & 0 & $\underline{0.1}$ & 0.3 & 0.5 & 0.8 & 0.5 & 0.8 & 1 & 1 & 0.8 & 1 & 1 & 1 \\
\hline 风险中性 & 0 & 0 & 0 & 0 & 0 & 0.1 & 0.2 & 0.3 & 0.3 & 0.5 & 0.8 & 1 & 0.5 & 0.8 & 1 & 1 \\
\hline 风险偏好 & 0 & 0 & 0 & 0 & 0 & 0 & 0 & 0 & 0 & 0.1 & 0.2 & 0.3 & 0.1 & 0.3 & 0.5 & 0.8 \\
\hline 中心风力 & \multicolumn{4}{|c|}{13 级 } & \multicolumn{4}{|c|}{14 级 } & \multicolumn{4}{|c|}{15 级 } & \multicolumn{4}{|c|}{$>15$ 级 } \\
\hline 提前时间 & 24 & 12 & 8 & 4 & 24 & 12 & 8 & 4 & 24 & 12 & 8 & 4 & 24 & 12 & 8 & 4 \\
\hline 风险厌恶 & 1 & 1 & 1 & 1 & 1 & 1 & 1 & 1 & 1 & 1 & 1 & 1 & 1 & 1 & 1 & 1 \\
\hline 风险中性 & 0.8 & 1 & 1 & 1 & 1 & 1 & 1 & 1 & 1 & 1 & 1 & 1 & 1 & 1 & 1 & 1 \\
\hline 风险偏好 & 0.3 & 0.5 & 0.8 & 1 & 0.5 & 0.8 & 1 & 1 & 0.8 & 1 & 1 & 1 & 1 & 1 & 1 & 1 \\
\hline
\end{tabular}

表 4. 根据当前位置风力渔船回港概率表

\begin{tabular}{c|cccccc}
\hline & \multicolumn{8}{c}{ 级 } & $\mathbf{8}$ 级 & $\mathbf{9}$ 级 & $\mathbf{1 0}$ 级 & $\mathbf{1 1}$ 级 & $>11$ 级 \\
\hline 风险厌恶 & 0 & $\underline{\mathbf{0 . 1}}$ & 0.5 & 1 & 1 & 1 \\
风险中性 & 0 & 0 & 0.1 & 0.8 & 0.5 & 1 \\
风险偏好 & 0 & 0 & 0 & 0.5 & 0.8 & 1 \\
\hline
\end{tabular}

表 4 给出了渔船根据当前所处位置的台风风力 $W_{i}$ 决定回港的概率 $P b_{i}$ 。 如表中带下画线的数字表 示，当渔船所处位置的风力为 8 级时，风险厌恶型 渔船选择回港的概率为 0.1 。

除对环境的响应之外, 渔船会和政府进行交互。 渔船“是否回港”决策会受到政府 “催促”回港次数 的影响, 其回港的概率 $P b_{i}$ 设定如表 5 所示。如表 中带下划线的数字表示，当政府催促回港 1 次时， 风险厌恶型渔船, 选择回港的概率为 0.5 。
此外渔船之间也会进行交互, 渔船会受到与自 身距离一定范围内的渔船的决策的影响。在影响半 径 $R$ 内，决定回港渔船的比例，会影响不同风险偏 好的渔船做出回港的决策, 回港概率 $P b_{i}$ 设定如表 6 所示。如表中带下划线的数字表明，对于风险厌恶 型渔船, 当影响半径内有 $0 \sim 10 \%$ 的渔船决定回港时, 它有 0.5 的概率可能选择回港。

表 5. 根据政府催促回港次数渔船回港概率表

\begin{tabular}{c|ccccc}
\hline & $\mathbf{1}$ 次 & $\mathbf{2}$ 次 & $\mathbf{3}$ 次 & $\mathbf{4}$ 次 & 5 次 \\
\hline 风险厌恶 & $\underline{\mathbf{0 . 5}}$ & 1 & 1 & 1 & 1 \\
风险中性 & 0.2 & 0.5 & 0.8 & 1 & 1 \\
风险偏好 & 0 & 0.2 & 0.5 & 0.8 & 1 \\
\hline
\end{tabular}

表 6. 根据影响半径内回港渔船比例 渔船回港概率表

\begin{tabular}{c|ccccc}
\hline & $\mathbf{0 ~ 1 0 \%}$ & $\mathbf{1 0 \%} \mathbf{3 0 \%}$ & $\mathbf{3 0 \%} \mathbf{5 0 \%}$ & $\mathbf{5 0 \%} \mathbf{8 0 \%}$ & $\mathbf{8 0 \%} \mathbf{1 0 0 \%}$ \\
\hline 风险厌恶 & $\mathbf{0 . 1}$ & 0.5 & 0.8 & 1 & 1 \\
风险中性 & 0 & 0.1 & 0.5 & 0.8 & 1 \\
风险偏好 & 0 & 0 & 0 & 0.1 & 0.3 \\
\hline
\end{tabular}

为使逻辑关系更容易处理, 认为渔船基于上述 四种因素的判断是独立的, 无论哪种因素使得渔船 做出“回港”的决定, 渔船最终都会回港。

（2）港口选择

对于所有的渔船，首选港口均为家乡港口 $H h$,
渔船根据估计得到的提前回到港口的时间，以一定 的概率选择就近避风, 决策概率 $P n_{i}$ 如表 7 。如表中 带下划线的数字 0.8 , 表示根据预计回港时间, 仅能 使渔船在容许回港时间前 2 小时回到港口时，风险 偏好型渔船选择就近避风的概率为 0.8 。 
表 7. 不同提前回港时间下渔船决定就近避风的概率

\begin{tabular}{|c|c|c|c|c|c|c|c|c|}
\hline & $\begin{array}{c}<0 \\
\text { 小时 }\end{array}$ & & $2-4$ & $4-8$ & $8-12$ & $12-16$ & $16-20$ & $\begin{array}{c}>20 \text { 小 } \\
\text { 时 }\end{array}$ \\
\hline 风险厌恶 & 1 & 1 & 1 & 0.8 & 0.5 & 0.3 & 0.1 & 0 \\
\hline 风险中性 & 1 & 1 & 0.8 & 0.5 & 0.3 & 0.1 & 0 & 0 \\
\hline 风险偏好 & 1 & $\underline{0.8}$ & 0.5 & 0.3 & 0.1 & 0 & 0 & 0 \\
\hline
\end{tabular}

\section{3 渔船回港个体自主决策仿真模型}

当气象部门发布台风预报信息时，确定了一个 渔船的容许回港时间 $T$, 政府部门的渔船回港避风 应急行动启动。根据当前时刻的台风中心实际风力、 台风大风预报、渔船位置状态, 运行仿真模型, 以 考察渔船回港时机和选择港口。

仿真模型的时间间隔为 1 小时，当所有渔船的 回港状态 $I b_{i}$ 变成 1 后, 完成 1 次仿真, 记录渔船回 港编号 $S h_{i}$ 和相应的渔船的回港时间 $T_{i}$ 。回港时间 为海上运行时间 $t_{i}$ 和延迟回港时间 $t w_{i}$ 的总和。

在每一时刻，对于每条回港状态为 0 的渔船, 进行一次是否回港决策。渔船依次按照政府催促回 港次数、影响半径内回港渔船比例、台风中心风力 和当前位置风力判断是否需要回港。一旦上述任意 条件得到满足, 渔船则做出回港的决策, 即渔船的 回港状态 $I b_{i}$ 变更为 1 , 并跳出是否回港判断。根据 渔船回到家乡港口的航行时间和当前时刻的延迟时 间，按照港口选择的概率表，判断渔船是选择回到 家乡港口还是就近港口, 记录渔船回港编号 $S h_{i}$, 和 回港时间 $T_{i}$ 。

在时刻末期，政府部门对所有渔船依次判断， 是否需要在下一时刻对其进行“催促回港”。对于所 有未回港的渔船, 计算其影响半径内回港渔船的比 例。

将整个仿真模型运行多次, 评估每一次仿真得 到回港方案的风险, 对所有仿真结果取平均值, 即 可评估渔船在个体自主回港状态下的风险情况。

\section{3. 浙江省台风渔船回港避风个体自主决策仿 真案例}

\section{1 数据处理与说明}

根据《浙江省沿海标准渔港布局与建设规划》 ${ }^{[15]}$, 截止到 2006 年底, 浙江省年共有海洋渔船 34506 艘, 各类渔港 208 座。计划在十年内, 将海洋机动 渔船数控制在 34500 艘左右; 到 2012 年底, 新增 有效避风港池面积 2000 万平方米，由 2006 年的
1650 万平方米增加到 3650 万平方米。

考虑到模型规模和电脑运行速度, 为说明问题, 并不失代表性，本示例假设有 3500 条渔船，前往 20 个不同港口避风。

\subsection{1港口数据}

根据《浙江省沿海标准渔港近期建设项目布局 图》 ${ }^{[15]}$, 浙江省在 2012 年重点建设一、二、三级 渔港 69 座, 图中还显示了国家级中心渔港和等级以 下渔港, 所有共计 81 座。在这 81 座港口中, 进行 分层随机抽样, 在中心渔港、一级渔港、二级渔港、 三级渔港和等级以下渔港中, 各选取 $2,4,5,3$, 6 个。

港口具有地理坐标、等级、避风面积、可容纳 渔船等级等属性，基本参数如表 8 所示:

- 港口的地理坐标通过数字化布局图时, 使用的 投影坐标得到。

- 港口的等级由布局图中的专题图符号可以看 出。

- 渔港的避风水域面积通过一定的合理假设产 生随机数模拟得到:

1) 基本保证所有 208 座渔港总避风面积在 3650 万平方米左右。

2）上一等级渔港避风面积是下一级的两倍左 右。

3）同等级渔港避风面积可以在一定范围内变 化。

- 港口的可容纳渔船等级根据渔港等级假设得 出。

\subsection{2 渔船数据}

根据 2010 年 9 月在江苏南通和 2011 年 6 月在 浙江岱山的关于渔船管理的调研，目前渔船上的信 息系统已基本配备完善，在台风来临时，海上需避 风渔船的地理位置及其他船只属性, 可以由管理部 门通过信息管理系统实时获取。

在本示例中, 待回港渔船采取在 ArcGIS 中生 成随机点的方式产生。假设浙江附近海域有 3500 只渔船需要回港避风, 其中有大型渔船 20 只, 在距 海岸线 200-300km 的范围内作业，中型渔船 1480 只, 距海岸线 130-200km, 小型渔船 2000 只, 距海 岸线 80-150km。根据《标准渔港设计规范》中的船 型尺度表，大型渔船代表船型选择“开创号”，中型 渔船代表船型选择 HG818 型渔船, 小型渔船选择 $10 \mathrm{kw}$ 木质渔船。相关参数如表 9 。 
表 8. 渔港参数表

\begin{tabular}{|c|c|c|c|c|}
\hline 编号 & 名称 & \multicolumn{2}{|c|}{ 坐标 $\mathbf{x}(\boldsymbol{m})$} & 坐标 $\mathbf{y}(m)$ \\
\hline 0 & 沥港渔港 & \multicolumn{2}{|c|}{1597857} & 3315665 \\
\hline 1 & 舟山中心渔港 & \multicolumn{2}{|c|}{1628658} & 3308746 \\
\hline 2 & 湖头渡渔港 & \multicolumn{2}{|c|}{1596071} & 3264441 \\
\hline 3 & 鸿屿渔港 & \multicolumn{2}{|c|}{1583684} & 3254174 \\
\hline 4 & 桐照渔港 & \multicolumn{2}{|c|}{1579555} & 3251607 \\
\hline 5 & 国庆渔港 & \multicolumn{2}{|c|}{1570627} & 3245581 \\
\hline 6 & 圆山渔港 & \multicolumn{2}{|c|}{1582903} & 3245135 \\
\hline 7 & 白歧渔港 & \multicolumn{2}{|c|}{1583684} & 3212659 \\
\hline 8 & 胡陈港渔港 & \multicolumn{2}{|c|}{1594174} & 3214222 \\
\hline 9 & 鹤浦渔港 & \multicolumn{2}{|c|}{1620846} & 3215003 \\
\hline 10 & 海游渔港 & \multicolumn{2}{|c|}{1579331} & 3202504 \\
\hline 11 & 路桥金清渔港 & \multicolumn{2}{|c|}{1600758} & 3139785 \\
\hline 12 & 坎门中心渔港 & \multicolumn{2}{|c|}{1578885} & 3085772 \\
\hline 13 & 杏湾渔港 & \multicolumn{2}{|c|}{1554445} & 3090682 \\
\hline 14 & 元觉花岗渔港 & \multicolumn{2}{|c|}{1570404} & 3064456 \\
\hline 15 & 兰田渔港 & \multicolumn{2}{|c|}{1539044} & 3066800 \\
\hline 16 & 西湾渔港 & \multicolumn{2}{|c|}{1527996} & 3028298 \\
\hline 17 & 炎亭渔港 & \multicolumn{2}{|c|}{1531121} & 3006202 \\
\hline 18 & 石砰渔港 & \multicolumn{2}{|c|}{1530898} & 3003077 \\
\hline 19 & 中墩渔港 & \multicolumn{2}{|c|}{1521188} & 2994595 \\
\hline \multicolumn{5}{|c|}{$\begin{array}{l}\text { 表 9. 船型参数表 (来源: SC/T 9010-2000 标准渔港设计 } \\
\text { 规范) }\end{array}$} \\
\hline 渔船类型 & 代表船型 & $\begin{array}{l}\text { 全长 } \\
(\mathrm{m})\end{array}$ & $\begin{array}{l}\text { 型宽 } \\
(\mathbf{m})\end{array}$ & $\begin{array}{c}\text { 船速 } \\
(\mathrm{km} / \mathrm{h})\end{array}$ \\
\hline 大 & 开创号 & 92 & 15 & 15 \\
\hline 中 & $\begin{array}{l}\text { HG818 型 } \\
\text { 渔船 }\end{array}$ & 31.88 & 6 & 10 \\
\hline 小 & $\begin{array}{l}10 \mathrm{kw} \text { 木 } \\
\text { 质渔船 }\end{array}$ & 7.59 & 3 & 5 \\
\hline
\end{tabular}

渔船“家乡港口”属性, 参考港口可接纳渔船类 型和港口面积因素, 按照渔船编号依次将大型渔船 平均分配到 0 级和 1 级港口, 将中型渔船平均分配 到 0 级- 3 级港口, 将小型渔船平均分配到 2 级-4 级 港口。对于“风险偏好”属性, 设定每条渔船属于风 险偏好型、风险中性型和风险厌恶型的概率为: 0.1 , 0.7，0.2。通过产生随机数, 得到每条渔船的风险偏 好值。

规定回港时间为 48 小时, 影响半径为: 50 千 米, 使用 2011 年 8 月 5 日的“台风梅花”的预警信息,

\begin{tabular}{|c|c|c|c|c|}
\hline \multirow{2}{*}{ 等级 } & \multirow{2}{*}{ 面积 $\left(m^{2}\right)$} & \multicolumn{3}{|c|}{ 可容纳船型 } \\
\hline & & 大 & 中 & 小 \\
\hline 二级渔港 & 172033.3 & 0 & 1 & 1 \\
\hline 中心渔港 & 492321.6 & 1 & 1 & 1 \\
\hline 等级以下渔港 & 26711.21 & 0 & 0 & 1 \\
\hline 等级以下渔港 & 35314.03 & 0 & 0 & 1 \\
\hline 一级渔港 & 321154.8 & 1 & 1 & 1 \\
\hline 二级渔港 & 155020.2 & 0 & 1 & 1 \\
\hline 三级渔港 & 72745.2 & 0 & 1 & 1 \\
\hline 等级以下渔港 & 47123.76 & 0 & 0 & 1 \\
\hline 二级渔港 & 162601.8 & 0 & 1 & 1 \\
\hline 一级渔港 & 374460 & 1 & 1 & 1 \\
\hline 等级以下渔港 & 45335.96 & 0 & 0 & 1 \\
\hline 一级渔港 & 311549.2 & 1 & 1 & 1 \\
\hline 中心渔港 & 665499.4 & 1 & 1 & 1 \\
\hline 二级渔港 & 154217.4 & 0 & 1 & 1 \\
\hline 等级以下渔港 & 22716.84 & 0 & 0 & 1 \\
\hline 三级渔港 & 69297.2 & 0 & 1 & 1 \\
\hline 三级渔港 & 74508.33 & 0 & 1 & 1 \\
\hline 一级渔港 & 328513.5 & 1 & 1 & 1 \\
\hline 二级渔港 & 176833.5 & 0 & 1 & 1 \\
\hline 等级以下渔港 & 36340.99 & 0 & 0 & 1 \\
\hline
\end{tabular}

台风梅花中心风力 14 级, 大风预报图为图 2 所示。 需做说明的是，图 1 为 24 小时的大风预报数据，理 论上应该在 8 月 5 日同时获得一张 24-48 小时大风 预报图。由于数据获取限制, 认为 48 小时内, 大风 的预报均为图 2。

使用 C\#语言编写程序，运行 5.3 中的仿真模型 200 次, 记录的渔船延迟回港时间、政府催促次数 和回港方案。

\section{2 模型运行结果}

\subsection{1 延迟回港结果}

在 200 次仿真过程中, 所有的 3500 只渔船, 共 有 652 只曾发生过延迟回港的现象, 占总渔船数量 的 $18.63 \%$ ，出现的最大延迟时间为 44 号渔船的 14 小时，计算每只渔船平均的延迟回港时间，该渔船 也具有最大的平均延迟回港时间，为 3.785 小时。 在 200 次仿真中, 渔船延迟回港结果的统计表 10 所示:

表 10. 200 次仿真延迟回港结果统计

\begin{tabular}{c|ccc|c}
\hline & $\begin{array}{c}\text { 延迟回港 } \\
\text { 船只数量 }\end{array}$ & $\begin{array}{c}\text { 最大延迟 } \\
\text { 回港时间 }\end{array}$ & $\begin{array}{c}\text { 所有渔船累积 } \\
\text { 延迟回港时间 }\end{array}$ & 有延迟渔船 200 次仿真平均延迟时间 \\
\hline 最大值 & 527 & 14 & 677 & 3.785 \\
最小值 & 482 & 4 & 577 & 0.005 \\
\hline 平均值 & $\mathbf{5 0 6}$ & $\mathbf{8 . 1 1}$ & $\mathbf{6 1 7 . 1 2 5}$ & $\mathbf{0 . 9 4 6 5}$ \\
\hline
\end{tabular}


考察渔船在仿真过程中回港的情况, 计算随仿 真时间的推移, 回港渔船数量的变化。计算在 200 次仿真中每一时刻已回港渔船的平均值, 如图 3 所 示 (大型渔船数量见副坐标轴)。

总体来看, 本次回港避风中, “延迟回港”的现 象不是很严重, 这主要是因为本次台风中心风力已 达 14 级, 多数的渔船都认为这是比较严重的状况, 希望自己能够提前到港的时间较宽裕。

\subsection{2 催促回港结果}

在 200 次仿真过程中, 所有的 3500 只渔船, 共 有 156 只渔船受到过政府的“催促回港”，占总渔船 数量的 $4.46 \%$, 出现的最大催促回港次数为 2670 号 渔船的 5 次。在 200 次仿真中, 渔船受到催促回港 结果的统计如表 11 所示:

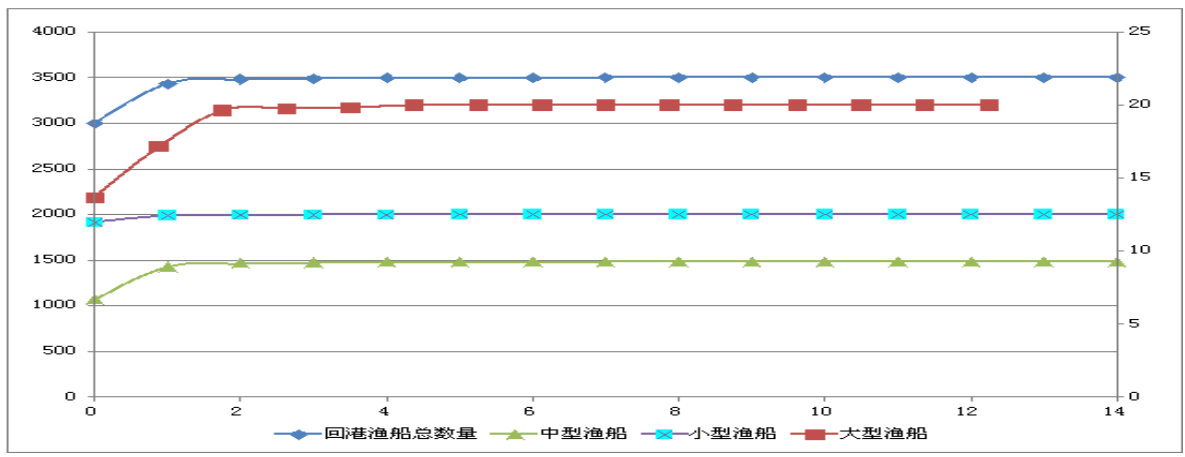

图 3. 仿真过程渔船回港情况

表 11.200 次仿真催促回港结果统计

\begin{tabular}{|c|c|c|c|c|}
\hline & $\begin{array}{l}\text { 催促回港 } \\
\text { 船只数量 }\end{array}$ & $\begin{array}{c}\text { 最大 } \\
\text { 催促回港次数 }\end{array}$ & $\begin{array}{l}\text { 所有渔船累积 } \\
\text { 催促回港次数 }\end{array}$ & $\begin{array}{c}\text { 有催促渔船 } 200 \text { 次仿真平均催 } \\
\text { 促次数 }\end{array}$ \\
\hline 最大值 & 115 & 5 & 97 & 0.925 \\
\hline 最小值 & 88 & 2 & 53 & 0.005 \\
\hline 平均值 & 101.64 & 3.54 & 76.645 & 0.4913 \\
\hline
\end{tabular}

\subsection{3 回港方案结果}

在本模型中, 渔船可能选择“回家避风”或“就近 避风”。从 200 次仿真的结果来看, 所有的 3500 只 渔船中, 有 1022 只渔船在所有的 200 次仿真中, 均 选择了“就近避风”, 说明它们在应急响应启动时, 所处的位置距家乡港口较远，在规定时间内很难安 全到达; 而有 1286 只渔船在 200 次仿真中, 均选择 了“回家避风”, 它们所处的位置恰好有充足的时间 安全回港。

\section{4. 结论和讨论}

本文从应急响应个体的自主决策行为出发, 设 计了渔船、政府、台风和港口的仿真模型，基于调 研了解的基本事实，建立了渔船回港的决策规则。 在仿真中, 通过对不同风险偏好渔船的“延迟回港” 决策和 “港口选择” 决策的逻辑规则设计，及政府 “催促回港” 决策设计，模拟了渔船在回港避风中
的延迟回港情况和港口选择情况。应急个体在自然 灾害中的自主应急行为和对管理部门应急安排的自 主执行, 将影响自然灾害应急响应的完成状况，因 此, 研究自然灾害应急响应个体的自主决策行为在 应急管理中有十分重要的意义。

本文通过仿真的方式模拟渔船在回港避风中的 自主决策行为, 在决策规则建立时, 仅根据调研的 基本事实进行了基于概率的逻辑设计，这使得模型 对于渔船自主回港决策行为的模拟只能反映基本情 况。在下一步的研究中, 应该对应急个体的风险认 知、风险识别和风险判断进行更为细致的研究, 得 到更为准确而符合实际意义的逻辑关系与参数, 建 立更为符合实际的应急个体在决策行为规则。

本文受国家自然科学基金 (41471424); 中央高 校基本科研业务费专项资金资助 


\section{参考文献}

[1] 周俊华. 西北太平洋热带气旋风险分析. 自然灾害 学报, 2004, 13(3): 146-151.

Zhou J H. Risk analysis of tropical cyclones in the northwestern Pacific. Journal of Natural Disasters, 2004, 13(3): 146-151.

[2] 中国气象局. 2011 年我国灾害性天气特点[EB/OL]. http://www.cma.gov.cn/2011zwxx/2011zyjgl/201203/t 20120305_163510.html

Characteristics of China's disastrous weather in 2011[EB/OL]. China Meteorological administration. http://www.cma.gov.cn/2011zwxx/2011zyjgl/201203/t 20120305_163510.html

[3] 冯华. 我国渔船数量世界第一。人民日报, 2012-3-26(2).

http://paper.people.com.cn/rmrb/html/2012-03/26/nw. D110000renmrb_20120326_8-02.htm

Feng $\mathrm{H}$. China fishing boats are the first in the world 。 People's Daily, 2012-3-26(2) http://paper.people.com.cn/rmrb/html/2012-03/26/nw. D110000renmrb_20120326_8-02.htm

[4] D. Helbing, I. Farkas, T. Vicsek Simulating dynamical features of escape panic. Nature, 407 (2000), pp. $487-490$

[5] Hao-Che $\mathrm{Wu}$, Michael K.Lindell, Carla S.Prater. Logistics of hurricane evacuation in Hurricanes Katrina and Rita. Transportation Research Part F, 2012, 15(4):445-461.

[6] 胡红，刘小明，杨孝宽. 基于最小安全间距的应急 交通疏散车辆跟驰模型. 北京工业大学学报, 2007, 33(10): 1070-1074.

Hu H, Liu X M, Yang X K. A Car-following Model of Emergency Evacuation Based on Minimum Safety Distance. Journal of Beijing University of Technology, 2007, 33(10): 1070-1074.

[7] $\mathrm{Tu}$ H Z, Tamminga Guus, Drolenga Hans, et al. Evacuation Plan of the City of Almere: Simulating the Impact of Driving Behavior on Evacuation Clearance Time. Proceedings of the 1st International Conference on Evacuation Modeling and Management, Netherlands, 2010: 67-75.

[8] Stern, E., Sinuany-Stern, Z., 1989. A behavioural-based simulation model for urban evacuation. Papers of the Regional Science Association 66, 87-103.

[9] Uchida, K., A model evaluating effect of disaster warning issuance conditions on "cry wolf syndrome" in the case of a landslide. European Journal of Operational Research 218 (2012) 530-537

[10] Railsback, S.F., Lytinen, S.L., Jackson, S.K., 2006. Agent-based simulation platforms: review and development recommendations. Simulation 82 (9), 609-623.

[11] Chen, X., Zhan, B.F., 2008. Agent-based modeling and simulation of urban evacuation: relative effectiveness of simultaneous and staged evacuation strategies. Journal of the Operational Research Society 59, 25-33.

[12] Banerjee, B., Abukmail, A., Kraemer, L., 2009. Layered intelligence for agent-based crowd simulation.

Simulation 85, 621-633.

doi:10.1177/0037549709340659.

[13] Magesh Nagarajan, Duncan Shaw, Pavel Albores Disseminating a warning message to evacuate: A simulation study of the behaviour of neighbours. European Journal of Operational Research 220 (2012) 810-819

[14] Hui, C., Goldberg, M., Magdon-Ismail, M., Wallace, W.A., 2008. Micro-simulation of diffusion of warnings. In: Fiedrich, F., Van de Walle, B. (Eds.), Proceedings of the 5th International ISCRAM Conference, Washington, DC, USA.

[15] 浙江省发展和改革委员会, 浙江省海洋与渔业局. 浙江省沿海标准渔港布局与建设规划 [EB/OL]. http://www.zjoaf.gov.cn/zfxxgk/ghjh/zxgh/2008/09/12/ ww092008091100008.shtml

Zhejiang Development and Reform Commission, Zhejiang ocean and Fishery Bureau. Zhejiang Province coastal port layout planning and construction standards[EB/OL].

http://www.zjoaf.gov.cn/zfxxgk/ghjh/zxgh/2008/09/12/ ww092008091100008.shtml 